Supplementary Material for

\title{
The Aarhus Chamber Campaign on Highly Oxidized Multifunctional Organic Molecules and Aerosols (ACCHA): Particle Formation and 5 Detailed Chemical Composition at Different Temperatures
}

Kasper Kristensen ${ }^{1}$, Louise N. Jensen ${ }^{2}$, Lauriane L. J. Quéléver ${ }^{3}$, Sigurd Christiansen ${ }^{2}$, Bernadette Rosati $^{2,4}$, Jonas Elm², Ricky Teiwes ${ }^{4}$, Henrik B. Pedersen ${ }^{4}$, Marianne Glasius ${ }^{2}$, Mikael Ehn ${ }^{3}$, Merete Bilde ${ }^{2}$ ${ }^{1}$ Department of Engineering, Aarhus University, 8000 Aarhus C, Denmark

${ }^{2}$ Department of Chemistry and iClimate, Aarhus University, 8000 Aarhus C, Denmark

$10{ }^{3}$ Institute for Atmospheric and Earth System Research - INAR / Physics, P.O. Box 64, FI-00014, University of Helsinki, Finland

${ }^{4}$ Department of Physics and Astronomy, Aarhus University, 8000 Aarhus C, Denmark

Correspondence to: Kasper Kristensen (kasper.kristensen@eng.au.dk) and Merete Bilde (bilde@chem.au.dk) 

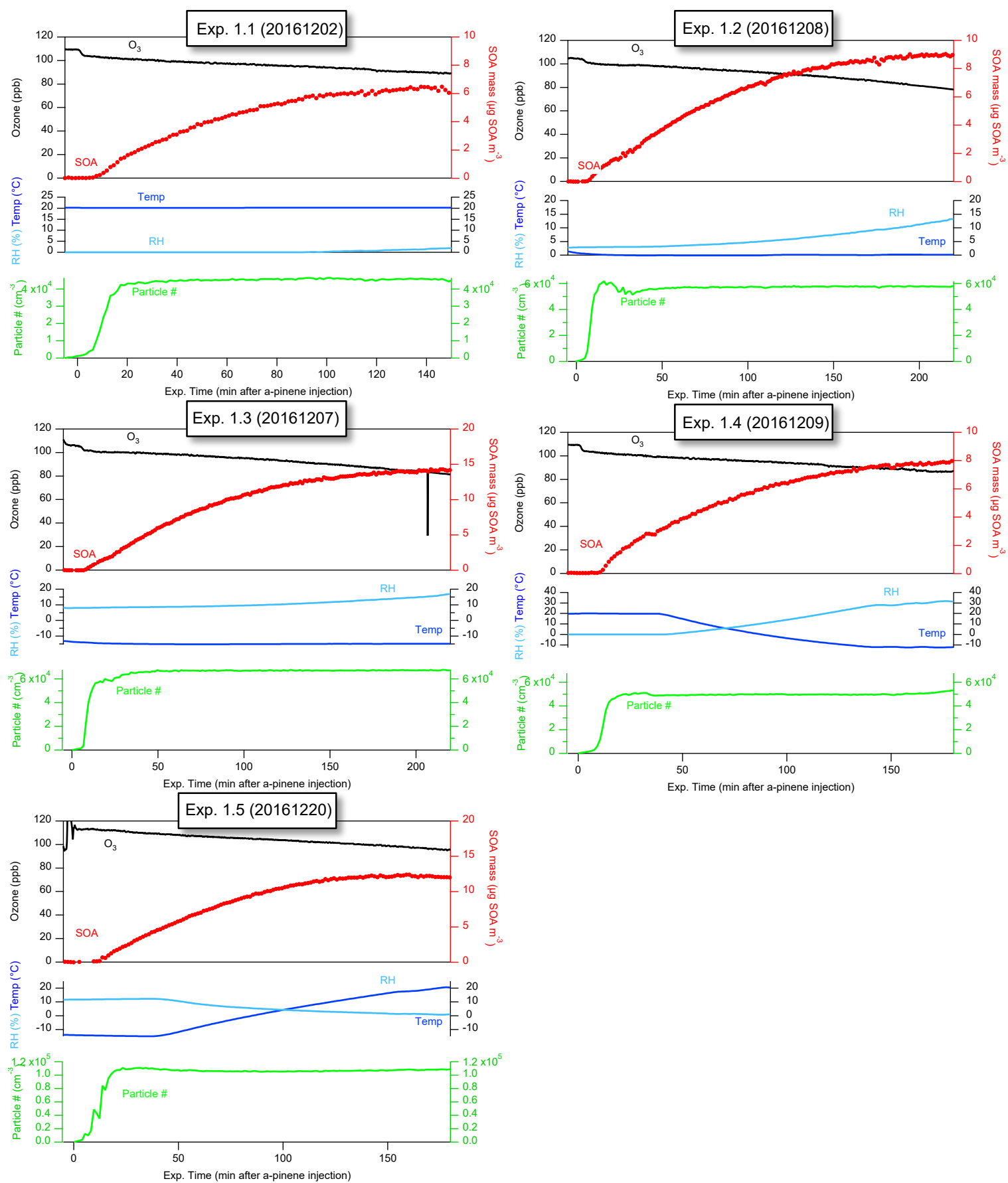

Exp. Time (min after a-pinene injection)

Figure S1. Concentration of $\mathrm{O}_{3}$ (ppb, black) and wall-loss corrected SOA mass $\left(\mu \mathrm{g} \mathrm{m}^{-3}\right.$, red) and particle number $\left(\mathrm{cm}^{-3}\right.$, green, particle diameter: $10-400 \mathrm{~nm}$, measured by SMPS) along with recorded RH $\left(\%\right.$, teal) and Temperature $\left({ }^{\circ} \mathrm{C}\right.$, blue) during 10 ppb $\alpha$-pinene oxidation experiments (Exp. 1.1-1.5) 

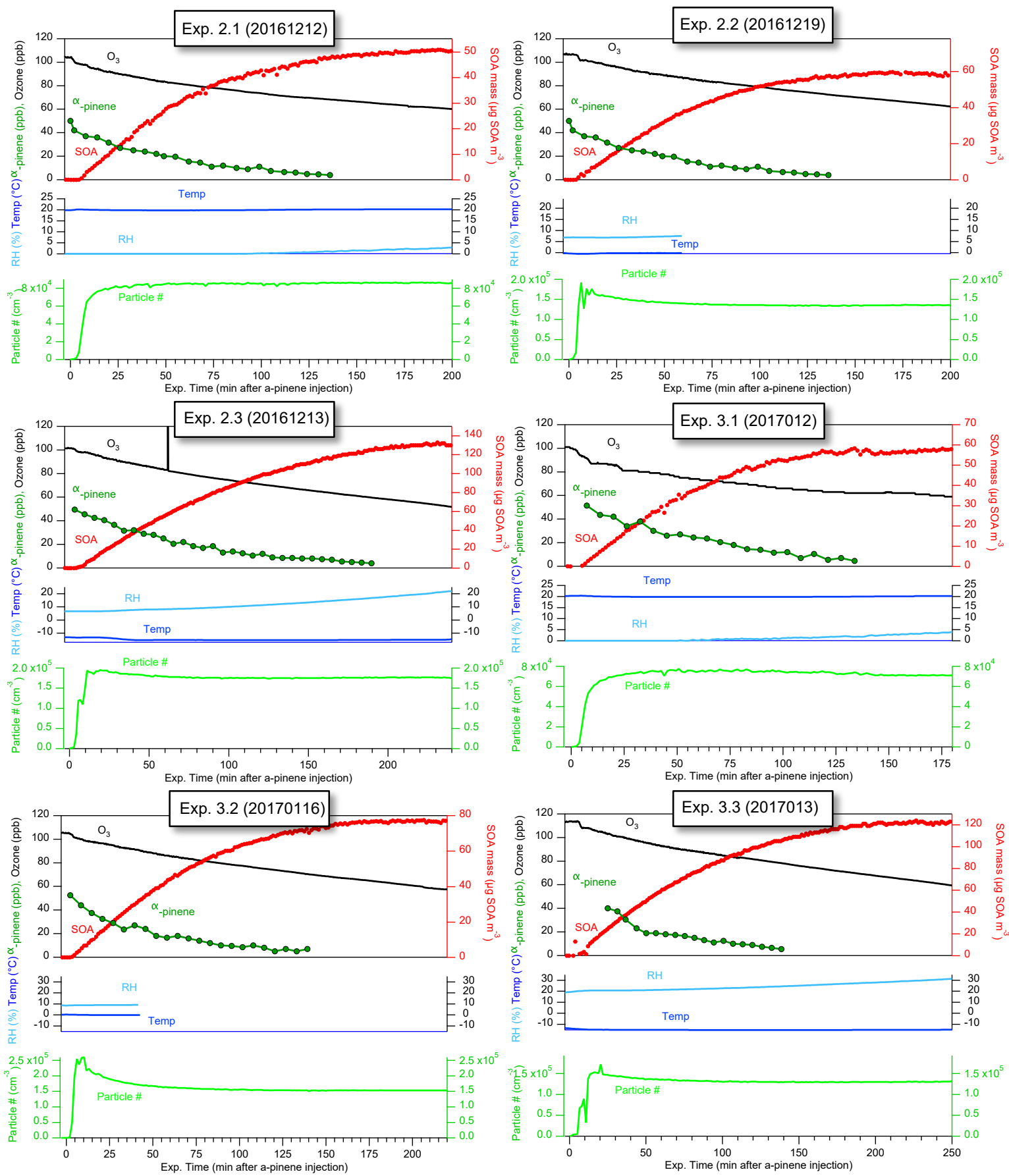

Figure S2. Concentration of $\mathrm{O}_{3}$ (ppb, black), $\alpha$-pinene (ppb, dark green), and wall-loss corrected SOA mass ( $\mu \mathrm{g} \mathrm{m}^{-3}$, red) and particle number ( $\mathrm{cm}^{-3}$, green, particle diameter: 10-400 nm, measured by SMPS) along with recorded $\mathrm{RH}(\%$, teal) and temperature $\left({ }^{\circ} \mathrm{C}\right.$, blue) during $50 \mathrm{ppb} \alpha$-pinene oxidation experiments (Exp. 2.1-2.3 (15 LMP VOC injection flow) \& 3.1-3.2 (30 LMP VOC injection flow)). 

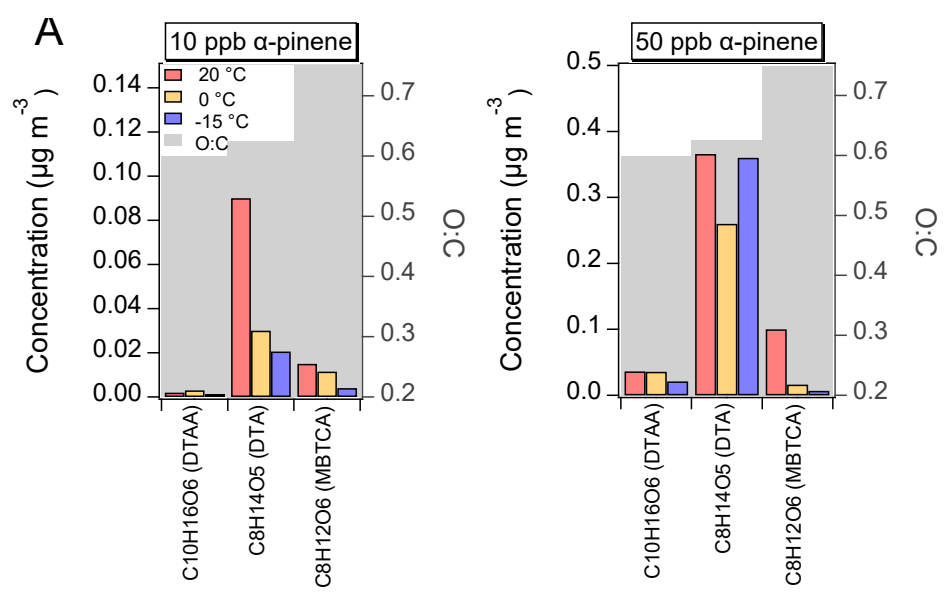

Figure S3. O:C-ratios and concentrations $\left(\mu \mathrm{g} \mathrm{m}^{-3}\right)$ of DTAA, DTA and MBTCA in SOA particles from 10 and $50 \mathrm{ppb} \alpha$-pinene oxidation experiments performed at 20,0 , and $-15^{\circ} \mathrm{C}$ (Exp. 1.1-1.3 and Exp. 2.1-2.3).

30 
Table S1. Chemical formula, molecular weight $\left(\mathrm{g} \mathrm{mol}^{-1}\right)$, and SOA particle concentration $\left(\mathrm{ng} \mathrm{m}^{-3}\right)$ of the identified organic acids and dimer esters in $10 \mathrm{ppb} \alpha$-pinene oxidation experiment (Exp. 1.1-1.5). Percentages increase (green) or decrease (red) in concentrations compared to concentrations found in experiment performed at $20^{\circ} \mathrm{C}($ Exp. 1.1) are shown.

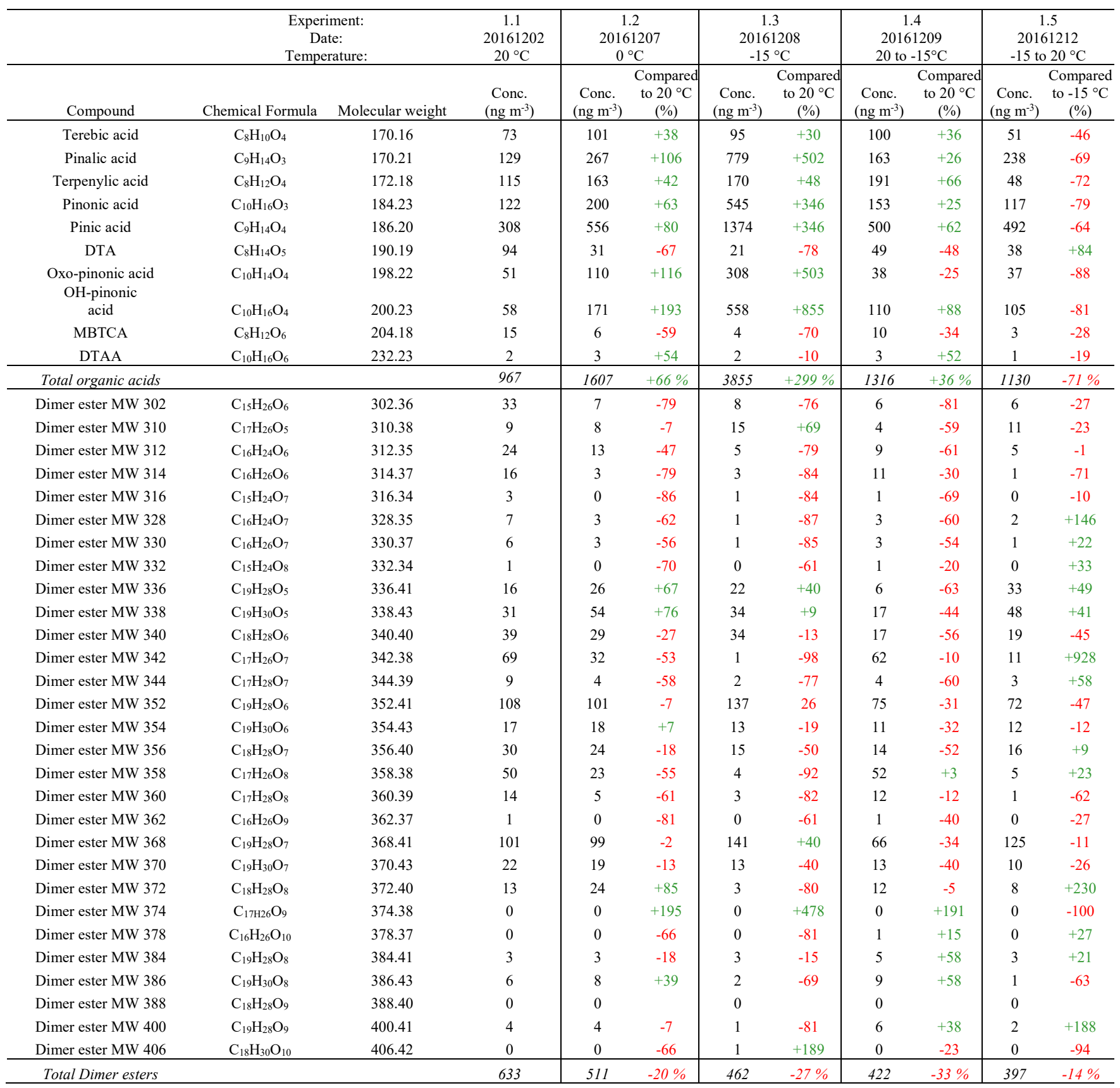


Table S2. Chemical formula, molecular weight $\left(\mathrm{g} \mathrm{mol}^{-1}\right)$, and SOA particle concentration ( $\mathrm{ng} \mathrm{m}^{-3}$ ) of the identified organic acids and 55 dimer esters in $50 \mathrm{ppb} \alpha$-pinene oxidation experiment (Exp. 2.1-2.3). Percentages increase (green) or decrease (red) in concentrations compared to concentrations found in experiment performed at $20^{\circ} \mathrm{C}$ (Exp. 1.1) are listed. Also, data from experiments with increased $\alpha$-pinene injection flow (Exp. 3.1 - 3.3) are shown.

\begin{tabular}{|c|c|c|c|c|c|c|c|c|c|c|}
\hline & \multicolumn{2}{|c|}{$\begin{array}{l}\text { Experiment: } \\
\text { Date: } \\
\text { Temperature: }\end{array}$} & $\begin{array}{c}2.1 \\
20161212 \\
20^{\circ} \mathrm{C} \\
\end{array}$ & \multicolumn{2}{|c|}{$\begin{array}{c}2.2 \\
20161219 \\
0^{\circ} \mathrm{C}\end{array}$} & \multicolumn{2}{|c|}{$\begin{array}{c}2.3 \\
20161213 \\
-15^{\circ} \mathrm{C}\end{array}$} & \multirow{2}{*}{$\begin{array}{c}3.1 \\
20170112 \\
20^{\circ} \mathrm{C} \\
\\
\text { Conc. } \\
\left(\mathrm{ng} \mathrm{m}^{-3}\right)\end{array}$} & \multirow{2}{*}{$\begin{array}{c}3.2 \\
20170116 \\
0^{\circ} \mathrm{C} \\
\\
\text { Conc. } \\
\left(\mathrm{ng} \mathrm{m}^{-3}\right) \\
\end{array}$} & \multirow{2}{*}{$\begin{array}{c}3.3 \\
20170113 \\
-15^{\circ} \mathrm{C} \\
\\
\text { Conc. } \\
\left(\text { ng m }^{-3}\right) \\
\end{array}$} \\
\hline Compound & Chemical Formula & Molecular weight & $\begin{array}{l}\text { Conc. } \\
\left(\mathrm{ng} \mathrm{m}^{-3}\right)\end{array}$ & $\begin{array}{l}\text { Conc. } \\
\left(\mathrm{ng} \mathrm{m}^{-3}\right)\end{array}$ & $\begin{array}{c}\text { Compared } \\
\text { to } 20^{\circ} \mathrm{C} \\
(\%)\end{array}$ & $\begin{array}{l}\text { Conc. } \\
\left(\mathrm{ng} \mathrm{m}^{-3}\right)\end{array}$ & $\begin{array}{c}\text { Compared } \\
\text { to } 20^{\circ} \mathrm{C} \\
(\%)\end{array}$ & & & \\
\hline Terebic acid & $\mathrm{C}_{8} \mathrm{H}_{10} \mathrm{O}_{4}$ & 170.16 & 668 & 807 & +38 & 503 & +30 & 604 & 357 & 272 \\
\hline Pinalic acid & $\mathrm{C}_{9} \mathrm{H}_{14} \mathrm{O}_{3}$ & 170.21 & 960 & 3294 & +106 & 3424 & +502 & 928 & 2009 & 2868 \\
\hline Terpenylic acid & $\mathrm{C}_{8} \mathrm{H}_{12} \mathrm{O}_{4}$ & 172.18 & 874 & 1366 & +42 & 1036 & +48 & 925 & 765 & 795 \\
\hline Pinonic acid & $\mathrm{C}_{10} \mathrm{H}_{16} \mathrm{O}_{3}$ & 184.23 & 538 & 3501 & +63 & 5030 & +346 & 518 & 2630 & 3983 \\
\hline Pinic acid & $\mathrm{C}_{9} \mathrm{H}_{14} \mathrm{O}_{4}$ & 186.20 & 1934 & 6861 & +80 & 8150 & +346 & 2022 & 4186 & 5923 \\
\hline DTA & $\mathrm{C}_{8} \mathrm{H}_{14} \mathrm{O}_{5}$ & 190.19 & 366 & 257 & -67 & 356 & -78 & 372 & 172 & 153 \\
\hline $\begin{array}{l}\text { Oxo-pinonic acid } \\
\text { OH-pinonic }\end{array}$ & $\mathrm{C}_{10} \mathrm{H}_{14} \mathrm{O}_{4}$ & 198.22 & 268 & 1184 & +116 & 1130 & +503 & 260 & 736 & 1040 \\
\hline & $\mathrm{C}_{10} \mathrm{H}_{16} \mathrm{O}_{4}$ & 200.23 & 493 & 2800 & +193 & 3615 & +855 & 492 & 1636 & 2367 \\
\hline MBTCA & $\mathrm{C}_{8} \mathrm{H}_{12} \mathrm{O}_{6}$ & 204.18 & 100 & 16 & -59 & 7 & -70 & 131 & 8 & 14 \\
\hline DTAA & $\mathrm{C}_{10} \mathrm{H}_{16} \mathrm{O}_{6}$ & 232.23 & 36 & 36 & +54 & 21 & -10 & 42 & 21 & 10 \\
\hline Dimer ester MW 302 & $\mathrm{C}_{15} \mathrm{H}_{26} \mathrm{O}_{6}$ & 302.36 & 8 & 52 & +589 & 22 & +187 & 17 & 39 & 15 \\
\hline Dimer ester MW 310 & $\mathrm{C}_{17} \mathrm{H}_{26} \mathrm{O}_{5}$ & 310.38 & 73 & 169 & +130 & 81 & +10 & 85 & 60 & 54 \\
\hline Dimer ester MW 312 & $\mathrm{C}_{16} \mathrm{H}_{24} \mathrm{O}_{6}$ & 312.35 & 143 & 77 & -46 & 19 & -87 & 133 & 23 & 17 \\
\hline Dimer ester MW 314 & $\mathrm{C}_{16} \mathrm{H}_{26} \mathrm{O}_{6}$ & 314.37 & 116 & 14 & -88 & 12 & -90 & 114 & 26 & 15 \\
\hline Dimer ester MW 316 & $\mathrm{C}_{15} \mathrm{H}_{24} \mathrm{O}_{7}$ & 316.34 & 19 & 5 & -75 & 2 & -87 & 19 & 2 & 1 \\
\hline Dimer ester MW 328 & $\mathrm{C}_{16} \mathrm{H}_{24} \mathrm{O}_{7}$ & 328.35 & 49 & 21 & -56 & 2 & -96 & 37 & 17 & 2 \\
\hline Dimer ester MW 330 & $\mathrm{C}_{16} \mathrm{H}_{26} \mathrm{O}_{7}$ & 330.37 & 49 & 22 & -54 & 27 & -45 & 65 & 10 & 6 \\
\hline Dimer ester MW 332 & $\mathrm{C}_{15} \mathrm{H}_{24} \mathrm{O}_{8}$ & 332.34 & 15 & 6 & -64 & 7 & -57 & 15 & 2 & 1 \\
\hline Dimer ester MW 336 & $\mathrm{C}_{19} \mathrm{H}_{28} \mathrm{O}_{5}$ & 336.41 & 86 & 410 & +379 & 189 & +121 & 121 & 288 & 143 \\
\hline Dimer ester MW 338 & $\mathrm{C}_{19} \mathrm{H}_{30} \mathrm{O}_{5}$ & 338.43 & 185 & 624 & +238 & 298 & +61 & 250 & 299 & 221 \\
\hline Dimer ester MW 340 & $\mathrm{C}_{18} \mathrm{H}_{28} \mathrm{O}_{6}$ & 340.40 & 129 & 223 & +73 & 265 & +105 & 104 & 104 & 157 \\
\hline Dimer ester MW 342 & $\mathrm{C}_{17} \mathrm{H}_{26} \mathrm{O}_{7}$ & 342.38 & 367 & 180 & -51 & 52 & -86 & 417 & 98 & 41 \\
\hline Dimer ester MW 344 & $\mathrm{C}_{17} \mathrm{H}_{28} \mathrm{O}_{7}$ & 344.39 & 55 & 53 & -3 & 29 & -46 & 52 & 28 & 19 \\
\hline Dimer ester MW 352 & $\mathrm{C}_{19} \mathrm{H}_{28} \mathrm{O}_{6}$ & 352.41 & 302 & 715 & +137 & 790 & +162 & 281 & 408 & 741 \\
\hline Dimer ester MW 354 & $\mathrm{C}_{19} \mathrm{H}_{30} \mathrm{O}_{6}$ & 354.43 & 75 & 257 & +243 & 201 & +168 & 82 & 139 & 148 \\
\hline Dimer ester MW 360 & $\mathrm{C}_{17} \mathrm{H}_{28} \mathrm{O}_{8}$ & 360.39 & 68 & 32 & -53 & 12 & -82 & 86 & 5 & 8 \\
\hline Dimer ester MW 362 & $\mathrm{C}_{16} \mathrm{H}_{26} \mathrm{O}_{9}$ & 362.37 & 10 & 2 & -80 & 2 & -78 & 14 & 3 & 3 \\
\hline Dimer ester MW 368 & $\mathrm{C}_{19} \mathrm{H}_{28} \mathrm{O}_{7}$ & 368.41 & 365 & 791 & +116 & 1049 & +187 & 440 & 423 & 1062 \\
\hline Dimer ester MW 370 & $\mathrm{C}_{19} \mathrm{H}_{30} \mathrm{O}_{7}$ & 370.43 & 104 & 193 & +86 & 111 & +7 & 133 & 166 & 84 \\
\hline Dimer ester MW 372 & $\mathrm{C}_{18} \mathrm{H}_{28} \mathrm{O}_{8}$ & 372.40 & 100 & 96 & -4 & 17 & -83 & 98 & 39 & 37 \\
\hline Dimer ester MW 374 & $\mathrm{C}_{17 \mathrm{H}_{26} \mathrm{O}_{9}}$ & 374.38 & 3 & 1 & -78 & 2 & -42 & 3 & 2 & 2 \\
\hline Dimer ester MW 378 & $\mathrm{C}_{16} \mathrm{H}_{26} \mathrm{O}_{10}$ & 378.37 & 5 & 0 & -92 & 1 & -88 & 8 & 0 & 1 \\
\hline Dimer ester MW 384 & $\mathrm{C}_{19} \mathrm{H}_{28} \mathrm{O}_{8}$ & 384.41 & 47 & 40 & -14 & 14 & -69 & 56 & 80 & 28 \\
\hline Dimer ester MW 386 & $\mathrm{C}_{19} \mathrm{H}_{30} \mathrm{O}_{8}$ & 386.43 & 75 & 93 & +25 & 10 & -86 & 122 & 86 & 80 \\
\hline Dimer ester MW 388 & $\mathrm{C}_{18} \mathrm{H}_{28} \mathrm{O}_{9}$ & 388.40 & 10 & 0 & -100 & 0 & -100 & 23 & 10 & 6 \\
\hline Dimer ester MW 400 & $\mathrm{C}_{19} \mathrm{H}_{28} \mathrm{O}_{9}$ & 400.41 & 26 & 15 & -41 & 1 & -98 & 70 & 129 & 36 \\
\hline Dimer ester MW 406 & $\mathrm{C}_{18} \mathrm{H}_{30} \mathrm{O}_{10}$ & 406.42 & 1 & 6 & +552 & 7 & +642 & 1 & 5 & 7 \\
\hline Total Dimer esters & & & 3073 & 4482 & $+46 \%$ & 3346 & $-9 \%$ & 3741 & 2661 & 3108 \\
\hline
\end{tabular}

